\title{
The impact of the lymph node density on overall survival in patients with Wilms' tumor: a SEER analysis
}

This article was published in the following Dove Press journal:

Cancer Management and Research

\author{
Haisheng You, ${ }^{1, *}$ Jin Yang, ${ }^{2,3, *}$ \\ Qingqing Liu, 2,3 Lina Tang,' \\ Qingting Bu, ${ }^{4}$ Zhenyu Pan, ${ }^{5}$ \\ Jun Lyu ${ }^{2,3}$
}

'Department of Pharmacy, The First Affiliated Hospital of Xi'an Jiaotong University, Xi'an, Shaanxi, People's Republic of China; ${ }^{2}$ Clinical Research Center, The First Affiliated Hospital of Xi'an Jiaotong University, Xi'an, Shaanxi, People's Republic of China; ${ }^{3}$ School of Public Health, Xi'an Jiaotong University Health Science Center, Xi'an, Shaanxi, People's Republic of China; ${ }^{4}$ Department of Genetics, Northwest Women's and Children's Hospital, Xi'an, Shaanxi, People's Republic of China; ${ }^{5}$ Department of Pharmacy, The Affiliated Children Hospital of Xi'an Jiaotong University, Xi'an, Shaanxi, People's Republic of China

*These authors contributed equally to this work

Correspondence: Jun Lyu

Clinical Research Center, The First

Affiliated Hospital of Xi'an Jiaotong

University, No. 277 West Yanta Road,

Xi'an, Shaanxi 71006I, People's Republic

of China

Tel +862985323614

Fax +862985323473

Email lujun2006@xjtu.edu.cn

\section{Zhenyu Pan}

Department of Pharmacy, The Affiliated Children Hospital of Xi'an Jiaotong University, No. 69 Xijuyuan Road, Xi'an, Shaanxi 710003, People's Republic of China

Tel +862987692188

Fax +862987692188

Email pzyxch@126.com
Objective: The objective of this study was to determine the impact of the lymph node density (LND) on overall survival of patients with Wilms' tumor (WT) using the Surveillance, Epidemiology, and End Results (SEER) database.

Methods: Data from the SEER database were extracted from patients with WT in whom the LND could be obtained. Patients were divided into a low LND group and high LND group. Survival curves based on the LND stratification were plotted using the Kaplan-Meier method and compared with the log-rank test. The impact of prognostic factors on overall survival was analyzed using Cox regression models.

Results: A total of 1,924 patients were identified from the database. Overall survival for the low LND group at 5, 10, and 20 years was significantly better than the high LND group (5-year survival: $94.1 \%$ vs $81.4 \%$; 10 -year survival: $92.6 \%$ vs $80.8 \%$; 20 -year survival: $90.6 \%$ vs $79.1 \%$; $P<0.001)$. In multivariate analysis, LND was a significant predictor of overall survival, regardless of whether it was a categorical variable or a continuous variable. Other significant predictors included age, race, SEER stage, and tumor laterality.

Conclusion: LND was a significant risk factor for overall survival of patients with WT. LND may provide a better prediction of the prognosis of WT patients and may be helpful for designing better treatments.

Keywords: Wilms' tumor, lymph node density, overall survival

\section{Introduction}

Wilms' tumor (WT) is the most common pediatric renal malignancy ${ }^{1}$ and affects approximately one child per 10,000 worldwide. ${ }^{2}$ The current research direction for WT is to limit the intensity of treatment without changing the prognosis in low-risk patients and to find more effective treatments for high-risk patients because of their significantly improved survival, from $30 \%$ in the 1930 s to more than $85 \%$ currently. ${ }^{3}$ More and better prognostic factors are needed for risk stratification of different patients. Using the WT histological type or stage as a stratification system of risk factors is no longer suitable for all cases. Some new prognostic factors, including age, tumor quality, ${ }^{3-5}$ loss of heterozygosity on chromosome $16 \mathrm{q}$ and $1 \mathrm{p},{ }^{6-9}$ Axl expression, ${ }^{10}$ and lymph node (LN) examination ${ }^{11}$ were found to affect the prognosis of patients with WT. In recent years, the lymph node ratio (LNR) or lymph node density (LND) has gained increasing attention in research on rectal cancer, ${ }^{12}$ colon cancer, ${ }^{13-16}$ and other cancers because it has been demonstrated to have a promising prognostic value. A recent study from Saltzman et $\mathrm{al}^{17}$ indicated that LND was significantly associated with overall survival in a favorable WT-based histology in 390 cases from the National 
Cancer Database during 2004-2013. However, more evidence is needed to further clarify whether LND is a prognostic factor for WT. The Surveillance, Epidemiology, and End Results database (SEER) database of the United States National Cancer Institute, which includes data from 1973 to 2014, is the largest registry of information on cancer incidence, mortality, survival, prevalence, and lifetime risk statistics in the United States. It includes 18 registries, which cover $\sim 28 \%$ of the population in the United States. ${ }^{18}$ The SEER database may be able to capture more WT cases.

The purpose of this study was to use the SEER database to determine the association between the LND and overall survival in WT.

\section{Methods}

\section{Patient selection}

Patient information was acquired from the SEER database, and this database was accessed using SEER*Stat version 8.3.4. ${ }^{19}$ We searched for patients by using the International Classification of Diseases for Oncology (ICD-O-3) histological diagnostic code 8960 for WT. We excluded patients whose LND could not be obtained. All data from the SEER database is freely available, and the projection of this study was approved by the institutional research committee of the First Affiliated Hospital of Xi'an Jiaotong University.

\section{Analytic variables}

Age, sex, race, number of LNs examined, SEER stage, tumor laterality, year of diagnosis, LND, follow-up time, and vital status were retrieved from the SEER database. The LND was defined as the number of positive LNs divided by the number of examined LNs.

\section{Statistical analysis}

Patients were stratified according to the LND in the low LND group and high LND group. The stratification cutoff was determined based on the Classification and Regression Tree. Patient characteristics between the two groups were compared. The normality of the continuous variables was evaluated. Continuous variables are expressed as the mean \pm SD conforming to the normal distribution and are otherwise expressed as the median (25th-75th percentile). Continuous variables that had a normal distribution were statistically compared with Student's $t$-test. Continuous variables that did not have the normal distribution were statistically compared with the Mann-Whitney $U$ test. The categorical variables were compared with Pearson's $\chi^{2}$ test. Survival curves based on the LND stratification were plotted using the Kaplan-Meier method and compared with the log-rank test. Cox regression models were used to analyze the relationship between the variables and overall survival. Variables with statistical significance in the univariate Cox regression models were analyzed in multivariate Cox regression models. The LND was analyzed as a categorical variable in the Cox regression models. Ten times the LND (TLND) was analyzed as a continuous variable in the Cox regression models. All statistics were assessed with SPSS version 24.0 (SPSS Inc., Chicago, IL, USA). A two-sided $P \leq 0.05$ was considered to be statistically significant.

\section{Results \\ Patient characteristics}

The patient characteristics are shown in Table 1. In total, 1924 WT patients with an LND were included in the study. The median age of the patients was 3 years $(25$ th -75 th percentile was 2-5). The proportion of female patients was slightly higher than males (52.3\% vs $47.7 \%)$. Most patients were white $(76.3 \%)$, had a unilateral tumor $(95.6 \%)$, were exposed to surgery (79.7\%), and were diagnosed from 2000 to $2014(71.5 \%)$. In the cohort, 832 patients had a localized tumor, 655 patients had a regional tumor, and 417 patients had a distant tumor. The median number of LN examined was 4 (25th-75th percentile was 2-7). The patient characteristics were stratified in different groups, namely, the low LND group and high LND group, applying 0.22 as cutoff value based on the Classification and Regression Tree. There were statistically significant differences between the two patient groups according to age $(P<0.001)$, number of LNs examined $(P<0.001)$, SEER stage $(P<0.001)$, and tumor laterality $(P=0.008)$.

\section{Survival analysis}

Representative Kaplan-Meier curves that were generated according to the LND stratification are shown in Figure 1. The overall survival for the low LND group at 5, 10, and 20 years was $94.1 \%, 92.6 \%$, and $90.6 \%$, respectively. The overall survival for the high LND group at 5, 10, and 20 years was $81.4 \%, 80.8 \%$, and $79.1 \%$, respectively. The logrank test showed that there were significant differences in overall survival between the low LND group and high LND group $(P<0.001)$.

Table 2 shows the risk factors related to survival according to Cox regression. We included age, sex, race, number of LNs examined, SEER stage, tumor laterality, year of diagnosis, and LND stratification to perform univariate Cox regression. Further multivariate Cox regression analysis 
Table I Patient characteristics in the study

\begin{tabular}{|c|c|c|c|c|}
\hline Variable & Total & Low LND & High LND & $P$-value \\
\hline Patients, $n$ & 1,924 & $1,64 \mid$ & 283 & \\
\hline Age (years), median (25th-75th percentile) & $3(2-5)$ & $3(2-5)$ & $4(3-6)$ & $<0.001$ \\
\hline Sex, n (\%) & & & & 0.529 \\
\hline Male & $917(47.7)$ & $787(48.0)$ & $130(45.9)$ & \\
\hline Female & $\mathrm{I}, 007(52.3)$ & $854(52.0)$ & $153(54.0)$ & \\
\hline Race, n (\%) & & & & 0.196 \\
\hline White & $\mathrm{I}, 468(76.3)$ & $\mathrm{I}, 240(75.6)$ & $228(80.6)$ & \\
\hline Black & $329(17.1)$ & $287(17.5)$ & $42(14.8)$ & \\
\hline Other & $105(5.5)$ & $94(5.7)$ & II (3.9) & \\
\hline LNs examined, median ( 25 th-75th percentile) & $4(2-7)$ & $4(2-8)$ & $3(2-5)$ & $<0.001$ \\
\hline SEER stage, $\mathrm{n}(\%)$ & & & & $<0.001$ \\
\hline Localized & $832(43.2)$ & $832(50.7)$ & $0(0.0)$ & \\
\hline Regional & $655(34.0)$ & $506(30.8)$ & $149(52.7)$ & \\
\hline Distant & $417(21.7)$ & $283(17.2)$ & $134(47.3)$ & \\
\hline Surgery, n (\%) & & & & 0.584 \\
\hline Yes & I,533 (79.7) & I,308 (79.7) & $225(79.5)$ & \\
\hline No & $6(0.3)$ & $6(0.4)$ & $0(0.0)$ & \\
\hline Unknown & $385(20.0)$ & $327(19.9)$ & $58(20.5)$ & \\
\hline Tumor laterality, $\mathrm{n}(\%)$ & & & & 0.008 \\
\hline Unilateral & $\mathrm{I}, 840(95.6)$ & $\mathrm{I}, 56 \mathrm{I}(95.1)$ & $279(98.6)$ & \\
\hline Bilateral & $84(4.4)$ & $80(4.9)$ & $4(1.4)$ & \\
\hline Year of diagnosis, n (\%) & & & & 0.161 \\
\hline 1988 & $24(1.2)$ & $22(1.3)$ & $2(0.7)$ & \\
\hline $1989-1997$ & $359(18.7)$ & $303(18.5)$ & $56(19.8)$ & \\
\hline $1998-2000$ & $166(8.6)$ & $133(8.1)$ & $33(11.7)$ & \\
\hline $2000-2014$ & I,375 (7I.5) & $\mathrm{I}, 183(72.1)$ & $192(67.8)$ & \\
\hline
\end{tabular}

Abbreviations: LNs, lymph nodes; LND, lymph node density; SEER, Surveillance, Epidemiology, and End Result.

showed that age (hazard ratio $[\mathrm{HR}]=1.049, P<0.001$ ), race (HR $=1.992$ for other vs white, $P=0.022$ ), SEER stage (HR $=2.893$ for distant vs localized, $P<0.001)$, tumor laterality $(\mathrm{HR}=2.014$ for bilateral vs unilateral, $P=0.021$ ), and LND stratification (HR $=2.102$ for high LND vs low LND, $P<0.001)$ were significant risk factors for poor overall survival.

We used the TLND (a continuous variable) instead of the LND stratification (a categorical variable) to analyze the risk factors according to the Cox regression (Table 3). In multivariate Cox regression, age (HR $=1.049, P<0.001)$, race (HR $=2.949$ for other vs white, $P=0.026$ ), SEER stage (HR $=2.965$ for distant vs localized, $P<0.001$ ), tumor laterality (HR $=1.998$ for bilateral vs unilateral, $P=0.023$ ), and TLND (HR $=1.098, P<0.001$ ) had a significant association with poor overall survival.

\section{Discussion}

In recent years, numerous studies have shown that LNR or LND is consistently associated with poor prognosis in various cancers, including cancers in the head and neck, ${ }^{20-23}$ rectal cancer, ${ }^{12}$ colon cancer, ${ }^{13-16,24}$ gastric cancer, ${ }^{25-29}$ pancreatic cancer, ${ }^{30}$ and the ampulla of Vater cancer. ${ }^{31}$ Furthermore, a study including 390 cases from the National Cancer Database published in 2017 suggested that LND might be a prognostic factor for favorable WT histology. ${ }^{17}$ However, the association of LND with the prognosis of WT patients still needs to be confirmed. Therefore, we utilized the SEER database to study the impact of LND on overall survival of patients with WT. In this study, we included 1,924 cases for whom the LND data were obtained from the SEER database; these cases were divided by LND into the low LND group and high LND group, with 0.22 as a cutoff point. The Kaplan-Meier curves showed that the high LND group had lower 5-, 10-, and 20-year survival rates compared to the low LND group based on the log-rank test. However, we found that low LND and high LND patients were not balanced with respect to age, number of LNs examined, SEER stage, and tumor laterality in the baseline comparison between the two groups. Therefore, all of the variables, including the LND stratification, were compared through univariate analysis and multivariate analysis using Cox regression to control the confounding factors. The results showed that age, race, SEER stage, tumor laterality, and LND stratification were significantly associated 


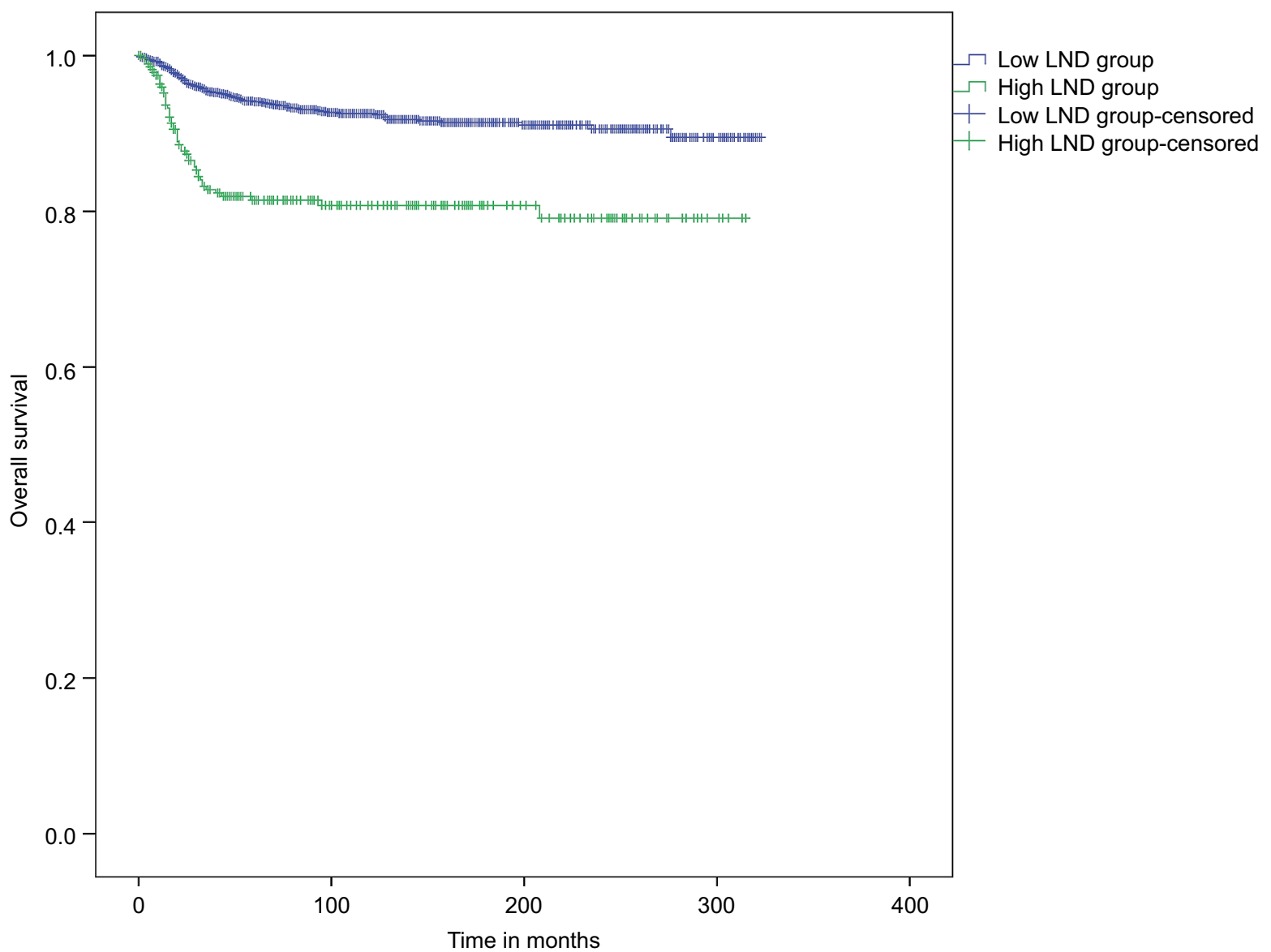

Figure I Kaplan-Meier curve for overall survival stratified by the LND.

Abbreviations: LND, lymph node density.

Table 2 Univariate and multivariate Cox regression analysis using the LND as a categorical variable

\begin{tabular}{|c|c|c|c|c|c|c|}
\hline \multirow[t]{2}{*}{ Variable } & \multicolumn{3}{|c|}{ Univariate analysis } & \multicolumn{3}{|c|}{ Multivariate analysis } \\
\hline & HR & $95 \% \mathrm{Cl}$ & $P$-value & HR & $95 \% \mathrm{Cl}$ & $P$-value \\
\hline Age & 1.045 & $1.03-1.06$ & $<0.001$ & 1.049 & $1.032-1.065$ & $<0.001$ \\
\hline \multicolumn{7}{|l|}{ Sex } \\
\hline Male & Reference & & & & & \\
\hline Female & 0.961 & $0.702-1.317$ & 0.806 & & & \\
\hline \multicolumn{7}{|l|}{ Race } \\
\hline White & Reference & & & Reference & & \\
\hline Black & 1.22 & $0.811-1.836$ & 0.34 & 1.268 & $0.842-1.91$ & 0.256 \\
\hline Other & 1.802 & $1.015-3.202$ & 0.044 & 1.992 & I.103-3.595 & 0.022 \\
\hline LNs examined & 0.984 & $0.954-1.015$ & 0.303 & & & \\
\hline \multicolumn{7}{|l|}{ SEER stage } \\
\hline Localized & Reference & & & Reference & & \\
\hline Regional & $\mathrm{I} .70 \mathrm{I}$ & $1.115-2.593$ & 0.014 & 1.467 & $0.939-2.29$ & 0.092 \\
\hline Distant & 3.839 & $2.578-5.717$ & $<0.001$ & 2.893 & $|.85-4.5| 4$ & $<0.001$ \\
\hline \multicolumn{7}{|l|}{ Tumor laterality } \\
\hline Unilateral & Reference & & & Reference & & \\
\hline Bilateral & 1.988 & $|| 27-3.5 \mid$. & 0.018 & 2.014 & $1.111-3.653$ & 0.021 \\
\hline \multicolumn{7}{|c|}{ Year of diagnosis } \\
\hline 1988 & Reference & & & & & \\
\hline $1989-1997$ & 2.836 & $0.387-20.778$ & 0.305 & & & \\
\hline $1998-2000$ & 3.798 & $0.503-28.656$ & 0.196 & & & \\
\hline $2000-2014$ & 3.156 & $0.434-22.957$ & 0.256 & & & \\
\hline \multicolumn{7}{|c|}{ LND stratification } \\
\hline Low LND & Reference & & & Reference & & \\
\hline High LND & 2.804 & $1.998-3.934$ & $<0.001$ & 2.102 & $1.44-3.07$ & $<0.001$ \\
\hline
\end{tabular}

Abbreviations: HR, hazard ratio; LNs, lymph nodes; LND, lymph node density; SEER, Surveillance, Epidemiology, and End Result. 
Table 3 Univariate and multivariate Cox regression analysis using the LND as a continuous variable

\begin{tabular}{|c|c|c|c|c|c|c|}
\hline \multirow[t]{2}{*}{ Variable } & \multicolumn{3}{|c|}{ Univariate analysis } & \multicolumn{3}{|c|}{ Multivariate analysis } \\
\hline & HR & $95 \% \mathrm{Cl}$ & $P$-value & HR & $95 \% \mathrm{Cl}$ & $P$-value \\
\hline Age & 1.045 & $1.03-1.06$ & $<0.001$ & 1.049 & $1.032-1.065$ & $<0.001$ \\
\hline \multicolumn{7}{|l|}{ Sex } \\
\hline Male & Reference & & & & & \\
\hline Female & 0.961 & $0.702-1.317$ & 0.806 & & & \\
\hline \multicolumn{7}{|l|}{ Race } \\
\hline White & Reference & & & Reference & & \\
\hline Black & 1.22 & $0.81 \mathrm{I}-1.836$ & 0.34 & 1.242 & $0.825-1.869$ & 0.299 \\
\hline Other & 1.802 & $1.015-3.202$ & 0.044 & 2.949 & $1.081-3.513$ & 0.026 \\
\hline LNs examined & 0.984 & $0.954-1.015$ & 0.303 & & & \\
\hline \multicolumn{7}{|l|}{ SEER stage } \\
\hline Localized & Reference & & & Reference & & \\
\hline Regional & $\mathrm{I} .70 \mathrm{I}$ & $1.115-2.593$ & 0.014 & 1.531 & $0.988-2.375$ & 0.057 \\
\hline Distant & 3.839 & $2.578-5.717$ & $<0.001$ & 2.965 & $1.907-4.612$ & $<0.001$ \\
\hline \multicolumn{7}{|c|}{ Tumor laterality } \\
\hline Unilateral & Reference & & & Reference & & \\
\hline Bilateral & 1.988 & $|| 27-3.5 \mid$. & 0.018 & 1.998 & $1.101-3.624$ & 0.023 \\
\hline \multicolumn{7}{|c|}{ Year of diagnosis } \\
\hline 1988 & Reference & & & & & \\
\hline 1989-1997 & 2.836 & $0.387-20.778$ & 0.305 & & & \\
\hline $1998-2000$ & 3.798 & $0.503-28.656$ & 0.196 & & & \\
\hline $2000-2014$ & 3.156 & $0.434-22.957$ & 0.256 & & & \\
\hline TLND & 1.143 & $1.095-1.193$ & $<0.001$ & 1.098 & $1.046-1.152$ & $<0.001$ \\
\hline
\end{tabular}

Abbreviations: HR, hazard ratio; LNs, lymph nodes; LND, lymph node density; SEER, Surveillance, Epidemiology, and End Result; TLND, ten times the LND.

with poor overall survival of WT patients. Although we used the Classification and Regression Tree method to determine a cutoff point of 0.22 and the study of Saltzman et $\mathrm{al}^{17}$ used 0.38 as the cutoff point, it was necessary to find the optimal LND cutoff for use in future clinical studies. To make the results more conservative, TLND as a continuous variable, instead of LND stratification, was included for reanalysis by Cox regression. Age, race, SEER stage, tumor laterality, and TLND, similarly, were significantly associated with poor overall survival. We included TLND instead of the LND. TLND ranges from 0 to 10 , while the LND ranges from 0 to 1. The HR for TLND in multivariate analysis was 1.098. This means that there was a $9.8 \%$ increase in the mortality risk if TLND increased by 1 , meaning that the LND increased by $10 \%$. Therefore, the specific meaning of HR can be determined by analyzing TLND instead of the LND.

Although we did not obtain chemotherapy and radiotherapy data from the SEER database, the year of diagnosis was categorized to represent treatment differences to some extent according to the publication years of the 2, 3, 4, and 5 trials of the National Wilms' Tumor Study Group (NWTS) (1981, 1989, 1998, 2001, respectively). ${ }^{5,32-34}$ The study of Ali et al ${ }^{35}$ showed WT patients diagnosed after 1981 (the publication year of NWTS 2) had better survival than those diagnosed before 1981, which may be due to the improvement of chemotherapy based on NWTS 2. The SEER database includes data from 1973 to 2014. Moreover, the earliest year of diagnosis in this study for the included patients was 1988 , and our study did not show stratification based on the year of diagnosis, which was significantly associated with survival for WT patients. This may be because patients who were diagnosed earlier than 1988 were excluded because they did not have LND data.

Theoretically, the number of LNs examined should be related to the WT prognosis because the examination of more LNs improves the accuracy and reliability of the tumor stage and stage impact of WT prognosis. The study of Zhuge et $\mathrm{al}^{11}$ from the SEER data and the Florida Cancer Data System found that the number of LNs examined is significantly associated with survival in WT. Moreover, our study did not find a significant association between the number of LNs and survival. This may be because of the following reasons: our study only included SEER data; we excluded patients whose number of LNs examined was 0 because the LND could not be obtained; and the number of LNs examined was analyzed as a continuous variable in this study rather than as a categorical variable, as in the study of Zhuge et al. ${ }^{11} \mathrm{In}$ addition, the results were supported by the study of Saltzman 
et al. ${ }^{17}$ Therefore, the relationship between the number of LNs and WT prognosis needs to be further clarified.

\section{The limitations in the study}

In the current study, there were still some limitations that need to be discussed. First, the baseline clinical characteristics in the low LND group and high LND group were not balanced regarding age, number of LNs examined, and SEER stage, and we did not adjust them to balance the data by propensity matching; therefore, the unadjusted Kaplan-Meier curve is shown in Figure 1. Second, 20\% of 1,924 cases did not have surgery data in the SEER data. It is generally accepted that a data loss of more than $20 \%$ is serious. We also could not identify the type of surgery in the remaining cases. Therefore, we did not include surgery data in the Cox regression analysis. However, it is necessary to analyze surgery data because surgery is an important treatment for WT and may be related to the LND. Third, the tumor staging system that the SEER database uses is the American Joint Committee on Cancer (AJCC) staging and SEER stage. Although AJCC staging is widely used in various tumors, there are no WT stages in AJCC staging. The WT staging system is mainly based on NWTS and International Society of Pediatric Oncology. $3,36,37$ Therefore, we can only use the SEER stage, which is rarely used in WT. Fourth, the chemotherapy and radiotherapy data were obtained from the SEER database, which were important prognostic factors for WT.

\section{Conclusion}

The current results show a significant association between the LND and overall survival in WT. Use of the LND may provide a better prediction of the prognosis of WT patients and guidance to adjust treatment. Further study is needed to research better treatment options based on prognostic factors to allow for a therapeutic reduction in low-risk patients and produce better survival in high-risk patients.

\section{Acknowledgments}

This study was supported by the National Social Science Foundation of China (No.16BGL183), the Natural Science Foundation of Shaanxi Province (No.2015JM8415), the Fundamental Research Funds for the Central Universities of China (No.2011jdhz55), the Clinical Research Award of the First Affiliated Hospital of Xi'an Jiaotong University of China (No.XJTU1AF-CRF-2016-021), and the Research Fund of Health Bureau of Xi'an (No.QFO1330).

\section{Disclosure}

The authors report no conflicts of interest in this work.

\section{References}

1. Birch JM, Breslow N. Epidemiologic features of Wilms tumor. Hematol Oncol Clin North Am. 1995;9(6):1157-1178.

2. Breslow N, Olshan A, Beckwith JB, Green DM. Epidemiology of Wilms tumor. Med Pediatr Oncol. 1993;21(3):172-181.

3. Metzger ML, Dome JS. Current therapy for Wilms' tumor. Oncologist. 2005;10(10):815-826.

4. Sredni ST, Gadd S, Huang CC, et al. Subsets of very low risk Wilms tumor show distinctive gene expression, histologic, and clinical features. Clin Cancer Res. 2009;15(22):6800-6809.

5. Green DM, Breslow NE, Beckwith JB, et al. Treatment with nephrectomy only for small, stage I/favorable histology Wilms' tumor: a report from the National Wilms' Tumor Study Group. J Clin Oncol. 2001;19(17):3719-3724.

6. Grundy PE, Breslow NE, Li S, et al. Loss of heterozygosity for chromosomes $16 \mathrm{q}$ and $\mathrm{lp}$ is an adverse prognostic factor in favorable histology Wilms tumor. A report from the National Wilms Tumor Study Group. J Clin Oncol. 2005;23(29):7312-7321.

7. Pan Z, He H, Tang L, et al. Loss of heterozygosity on chromosome 16q increases relapse risk in Wilms' tumor: a meta-analysis. Oncotarget. 2017;8(39):66467-66475

8. Dome JS, Fernandez CV, Mullen EA, et al. Children's Oncology Group's 2013 blueprint for research: renal tumors. Pediatr Blood Cancer. 2013;60(6):994-1000.

9. Pritchard-Jones K, Bergeron C, de Camargo B, et al. Omission of doxorubicin from the treatment of stage II-III, intermediate-risk Wilms' tumour (SIOP WT 2001): an open-label, non-inferiority, randomised controlled trial. Lancet. 2015;386(9999):1156-1164.

10. Zhu S, Liu G, Fu W, Hu J, Fu K, Jia W. Axl promotes the proliferation, invasion and migration of Wilms' tumor and can be used as a prognostic factor. Onco Targets Ther. 2017;10:955-963.

11. Zhuge Y, Cheung MC, Yang R, Koniaris LG, Neville HL, Sola JE. Improved survival with lymph node sampling in Wilms tumor. J Surg Res. 2011;167(2):e199-e203.

12. Li QG, Li DW, Zhuo CH, Cai GX, Cai SJ. Metastatic lymph node ratio can further stratify prognosis in rectal cancer patients treated with preoperative radiotherapy: a population-based analysis. Tumour Biol. 2014;35(7):6389-6395.

13. Berger AC, Sigurdson ER, LeVoyer T, et al. Colon cancer survival is associated with decreasing ratio of metastatic to examined lymph nodes. J Clin Oncol. 2005;23(24):8706-8712.

14. Park IJ, Choi GS, Jun SH. Nodal stage of stage iii colon cancer: the impact of metastatic lymph node ratio. J Surg Oncol. 2009;100(3):240-243.

15. Peschaud F, Benoist $\mathrm{S}$, Julie $\mathrm{C}$, et al. The ratio of metastatic to examined lymph nodes is a powerful independent prognostic factor in rectal cancer. Ann Surg. 2008;248(6):1067-1073.

16. Rosenberg R, Friederichs J, Schuster T, et al. Prognosis of patients with colorectal cancer is associated with lymph node ratio: a singlecenter analysis of 3,026 patients over a 25-year time period. Ann Surg. 2008;248(6):968-978.

17. Saltzman AF, Carrasco A Jr, Amini A, et al. Patterns of lymph node sampling and the impact of lymph node density in favorable histology Wilms tumor: an analysis of the national cancer database. $J$ Pediatr Urol. Epub 2017 Oct 31. pii: S1477-5131(17):30436-30439.

18. Wang S, Gao WC, Chen SS, et al. Primary site surgery for metastatic adrenocortical carcinoma improves survival outcomes: an analysis of a population-based database. Onco Targets Ther. 2017;10:5311-5315.

19. Surveillance, Epidemiology, and End Results (SEER) Program. SEER*Stat database: incidence-SEER 18 Regs research data + Hurricane Katrina impacted Louisiana cases, Nov 2016 Sub (1973-2014)-linked to county attributes-Total U.S., 1969-2015 counties. National Cancer Institute, DCCPS, Surveillance Research Program, Surveillance Systems Branch, released April 2017, based on the November 2016 submission.

20. Ebrahimi A, Clark JR, Zhang WJ, et al. Lymph node ratio as an independent prognostic factor in oral squamous cell carcinoma. Head Neck. 2011;33(9):1245-1251. 
21. Hong HR, Roh JL, Cho KJ, Choi SH, Nam SY, Kim SY. Prognostic value of lymph node density in high-grade salivary gland cancers. $J$ Surg Oncol. 2015;111(6):784-789.

22. Ong W, Zhao R, Lui B, et al. Prognostic significance of lymph node density in squamous cell carcinoma of the tongue. Head Neck. 2016;38(Suppl 1): e859-e866.

23. Hung YS, Chang SC, Liu KH, et al. A prognostic model based on lymph node metastatic ratio for predicting survival outcome in gastric cancer patients with N3b subclassification. Asian J Surg. Epub 2017 Dec 13. pii: S1015-S9584(17)30493-30501.

24. Amri R, Klos CL, Bordeianou L, Berger DL. The prognostic value of lymph node ratio in colon cancer is independent of resection length. Am J Surg. 2016;212(2):251-257.

25. Marchet A, Mocellin S, Ambrosi A, et al. The ratio between metastatic and examined lymph nodes ( $\mathrm{N}$ ratio) is an independent prognostic factor in gastric cancer regardless of the type of lymphadenectomy: results from an Italian multicentric study in 1853 patients. Ann Surg. 2007;245(4):543-552.

26. Wang $\mathrm{W}, \mathrm{Xu} \mathrm{DZ}, \mathrm{Li}$ YF, et al. Tumor-ratio e metastasis staging system as an alternative to the 7 th edition UICC TNM system in gastric cancer after D2 resection e results of a singleinstitution study of 1343 Chinese patients. Ann Oncol. 2011;22(9):2049-2056.

27. Wang J, Dang P, Raut CP, et al. Comparison of a lymph node ratio-based staging system with the 7th AJCC system for gastric cancer: analysis of 18,043 patients from the SEER database. Ann Surg. 2012;255(3): $478-485$.

28. Xu DZ, Geng QR, Long ZJ, et al. Positive lymph node ratio is an independent prognostic factor in gastric cancer after $\mathrm{d} 2$ resection regardless of the examined number of lymph nodes. Ann Surg Oncol. 2009;16(2):319-326.
29. Sun Z, Zhu GL, Lu C, et al. The impact of N-ratio in minimizing stage migration phenomenon in gastric cancer patients with insufficient number or level of lymph node retrieved: results from a Chinese monoinstitutional study in 2159 patients. Ann Oncol. 2009;20(5):897-905.

30. Dasari BV, Roberts KJ, Hodson J, et al. A model to predict survival following pancreaticoduodenectomy for malignancy based on tumour site, stage and lymph node ratio. HPB (Oxford). 2016;18(4): 332-338.

31. Dasari BV, Roberts KJ, Hodson J, et al. Prognostic value of the metastatic lymph node ratio in patients with resectable carcinoma of ampulla of vater. Medicine (Baltimore). 2015;94(42):e1859.

32. D'Angio GJ, Evans A, Breslow N, et al. The treatment of Wilms' tumor: results of the Second National Wilms' Tumor Study. Cancer. 1981;47(9):2302-2311.

33. D'Angio GJ, Breslow N, Beckwith JB, et al. Treatment of Wilms' tumor. Results of the Third National Wilms' Tumor Study. Cancer. 1989;64(2):349-360.

34. Green DM, Breslow NE, Beckwith JB, et al. Comparison between singledose and divided-dose administration of dactinomyein and doxorttbiein for patients with Wilms' tumor: a report from the National Wilms' Tumor Study Group. J Clin Oncol. 1998;16(1):237-245.

35. Ali AN, Diaz R, Shu HK, Paulino AC, Esiashvili N. A Surveillance, Epidemiology and End Results (SEER) program comparison of adult and pediatric Wilms' tumor. Cancer. 2012;118(9):2541-2551.

36. Vujani GM, Sandstedt B, Harms D, et al. Revised International Society of Paediatric Oncology (SIOP) working classification of renal tumors of childhood. Med Pediatr Oncol. 2002;38(2):79-82.

37. Kalapurakal JA, Dome JS, Perlman EJ, et al. Management of Wilms' tumour: current practice and future goals. Lancet Oncol. 2004;5(1):37-46.
Cancer Management and Research

\section{Publish your work in this journal}

Cancer Management and Research is an international, peer-reviewed open access journal focusing on cancer research and the optimal use of preventative and integrated treatment interventions to achieve improved outcomes, enhanced survival and quality of life for the cancer patient The manuscript management system is completely online and includes

\section{Dovepress}

a very quick and fair peer-review system, which is all easy to use. Visit http://www.dovepress.com/testimonials.php to read real quotes from published authors. 